\title{
Detección de Parásitos en Peces Marinos Destinados al Consumo Humano en Lima Metropolitana
}

\author{
Detection of Parasites in Marine Fish for Human Consumption in Lima \\ Enrique Serrano-Martínez ${ }^{1,2}$, Marco Quispe H. ${ }^{1}$, Elizabeth Hinostroza M. ${ }^{1}$, \\ Lucy Plasencia P. $^{1}$
}

\section{Resumen}

El presente estudio tuvo como objetivo evaluar la presencia de parásitos en cinco especies de peces marinos (Sarda chiliensis chiliensis [bonito], Scomber japonicus peruanus [caballa], Trachurus picturatus murphyi [jurel], Mugil cephalus [lisa] y Coryphaena hippurus [perico]) destinados al consumo humano. Se utilizaron 150 peces (30 por especie) procedentes de los terminales pesqueros de Chorrillos y Villa María del Triunfo en la provincia de Lima, Perú, entre enero y marzo de 2014. Se encontraron 13 especies de parásitos: monogeneos (Monocotile sp), digeneos (Dinurus sp), protozoos (Henneguya sp), acantocéfalo (Rhadinorhynchus sp), cestodos (Diphyllobothrium pacificum, Hepatoxylon trichiuri, Tentacularia coryphaenae, Nybelinia sp), nematodos (Anisakis simplex, Anisakis physeteris, Contracaecum sp, Proleptus sp) y artrópodos (Caligus sp). El 61.3\% de los especímenes presentaron al menos un parásito, habiendo una mayor prevalencia en lisa (86.7\%), seguida de perico (76.7\%). Asimismo, la mayor carga parasitaria se observó en lisa (424) y bonito (376). Los parásitos más frecuentes fueron Hepatoxylon trichiuri, Nybelinia sp y Proleptus sp. Asimismo, se encontraron parásitos de importancia en salud pública, como el Anisakis sp en todas las especies, a excepción de la lisa, y del Diphyllobothrium pacificum en bonito y perico.

Palabras clave: parásitos, anisakiosis, diphyllobothriosis, salud pública, inocuidad

\section{Abstract}

The aim of this study was to evaluate the presence of parasites in five species of marine fish (Sarda chiliensis chiliensis [Eastern Pacific bonito], Scomber japonicus peruanus [Pacific chub mackerel], Trachurus picturatus murphyi [Chilean jack mackerel],

\footnotetext{
${ }^{1}$ Grupo SALUVET-UPCH, Facultad de Medicina Veterinaria y Zootecnia, Universidad Peruana Cayetano Heredia, Lima, Perú

${ }^{2}$ E-mail: enrique.serrano@upch.pe
}

Recibido: 17 de mayo de 2016

Aceptado para publicación: 12 de octubre de 2016 


\begin{abstract}
Mugil cephalus [flathead grey mullet], and Coryphaena hippurus [common dolphinfish]) intended for human consumption. A total of 150 fish were used (30 per species) from the fishing terminals of Chorrillos and Villa María del Triunfo in the province of Lima, Peru, between January to March 2014. It was found 13 species of parasites: monogeneans (Monocotile sp), digenea (Dinurus sp), protozoa (Henneguya sp), acanthocephalan (Rhadinorhynchus sp), cestoda (Diphyllobothrium pacificum, Hepatoxylon trichiuri, Tentacularia coryphaenae, Nybelinia sp), nematoda (Anisakis simplex, Anisakis physeteris, Contracaecum sp, Proleptus sp) and arthropods (Caligus sp). The results showed that $61.3 \%$ of the specimens had at least one parasite, having the flathead grey mullet a higher prevalence (86.7\%), followed by the common dolphinfish (76.7\%). Similarly, greater parasite load was observed in flathead grey mullet (424) and Eastern Pacific bonito (376). The most common parasites were Hepatoxylon trichiuri, Nybelinia sp and Proleptus sp. Parasites of public health importance were found shuch as Anisakis sp in all species except for the flathead grey mullet and Diphyllobothrium pacificum in Eastern Pacific bonito and common dolphinfish.
\end{abstract}

Key words: parasites, anisakiasis, diphyllobothriasis, public health, food safety

\section{INTRODUCCIÓN}

En el Perú, el sector pesquero es la más importante fuente generadora de divisas después de la minería, destacándose la pesca marítima y en menor grado la continental (FAO, 2010). Es así que los volúmenes de desembarque en recursos hidrobiológicos marítimos en 2013 fueron de 5859000 toneladas métricas brutas (TMB), mientras que en los continentales fueron de $56500 \mathrm{TMB}$ (INEI, 2014).

El 19.8\% de la extracción de los recursos hidrobiológicos marítimos fue destinado al consumo humano directo (enlatado, congelado, curado o fresco), en tanto que el $80.2 \%$ fue destinado al consumo indirecto (harina y aceite de pescado) en 2013 (INEI, 2014); sin embargo, sea cual fuere su destino, existen requerimientos sanitarios nacionales e internacionales para asegurar la inocuidad de estos productos.

Estudios realizados a nivel nacional e internacional demuestran la presencia de especies parasitarias en los recursos hidrobiológicos (Pérez et al., 1999; Llerena et al., 2001; Cabrera et al., 2002; Iannacone,
2004; Aragort 2006; Iannacone y Alvariño, 2009; Vásquez-Ruiz y Jara-Campos, 2012; Chero et al., 2014). El parasitismo en peces ocasiona pérdidas socioeconómicas, ya sea por la transmisión al humano de patógenos de importancia en salud pública como el Anisakis sp y Diphyllobotrhium pacificum, o aquellas que, al estar presentes, pueden ocasionar el deterioro o daño del producto, el cual debe ser descartado de las líneas de comercio o producción, según lo establece la normativa sanitaria vigente.

Un ejemplo de ello es la normativa peruana que establece consideraciones sanitarias durante las operaciones de procesamiento del pescado fresco previo a la congelación y del pescado que será sometido al proceso de curado. Una de estas indica que el pescado que se encuentre parasitado se descartará de la línea de proceso (Decreto Supremo $\mathrm{N}^{\circ}$ 040-2001-PE).

Es de interés nacional mantener actualizada la data de la fauna parasitaria presente en las especies hidrobiológicas destinadas al consumo humano directa o indirectamente, con la finalidad de establecer programas sanitarios y recomendaciones para garantizar la inocuidad de estos pro- 
ductos. En tal sentido, el objetivo del presente estudio fue evaluar la presencia de parásitos en cinco especies de peces marinos destinados al consumo humano directo.

\section{Materiales y Métodos}

El estudio se llevó a cabo en los terminales pesqueros de Chorrillos y Villa María del Triunfo, localizados ambos en la provincia y departamento de Lima. Los análisis parasitológicos fueron realizados en el Laboratorio de Parasitología de la Facultad de Medicina Veterinaria y Zootecnia (FAVEZ) de la Universidad Peruana Cayetano Heredia (UPCH), Lima, Perú.

Se consideraron 30 especímenes por cada una de las cinco especies de peces marinos que entre enero y marzo de 2014 registraron mayores ingresos y comercio en los terminales pesqueros en estudio, como fueron: Sarda chiliensis chiliensis (bonito), Scomber japonicus (caballa), Trachurus symmetricus murphyi (jurel), Mugil cephalus (lisa) y Coryphaena hippurus (perico).

Se revisó la cavidad abdominal, la superficie de las vísceras y la vejiga natatoria en busca de helmintos (Adis et al., 2006). E1 estómago e intestino fueron colocados en placas de Petri con agua corriente para el examen del contenido previa disección. Los parásitos obtenidos se lavaron en solución fisiológica y se fijaron en formol al 10\% (cestodos) o alcohol de $70 \%$ caliente (nematodos, artrópodos y otros grupos parasitarios). La identificación de los cestodos (Schmidt, 1986; Khalil et al., 1994) se hizo después de la coloración con carmín de Semichon y la de los nematodos luego de la clarificación en solución de alcohol-fenol (Anderson et al., 2009). Después de la identificación de los trematodos según Adis et al. (2006), estos se lavaron y fijaron en formol al 10\% previo aplanamiento entre lámina y laminilla por 24 horas, para finalmente colorearlos con carmín acético de Semichon, deshidratarlos, clarificarlos y montarlos en preparaciones permanentes con bálsamo de Canadá (Eiras et al., 2002).

Las muestras de ectoparásitos fueron identificadas de acuerdo a Adis et al. (2006), observando presencia de lesiones o alguna especie parasitaria en piel y branquias. Se realizó un raspado en la piel y los frotis fueron observados en el estereoscopio a $4 x$ y en el microscopio óptico a 40x.

Los hallazgos se documentaron fotográficamente y el material se encuentra archivado en el Laboratorio de Parasitología de la FAVEZ-UPCH. Los valores de prevalencia se calcularon según Bush et al. (1997). El nivel de parasitismo de cada especie se determinó de acuerdo a los índices ecológicos sugeridos por Margolis et al. (1982) y Bush et al. (1997).

\section{REsultados}

Se observó la presencia de 13 especies de parásitos en las cinco especies de peces: monogeneos (Monocotile sp), digeneos (Dinurus sp), protozoos (Henneguya sp), acantocéfalo (Rhadinorhynchus sp), cestodos (Diphyllobothrium pacificum, Hepatoxylon trichiuri, Tentacularia coryphaenae, Nybelinia sp), nematodos (Anisakis simplex, Anisakis physeteris, Contracaecum sp, Proleptus sp) y artrópodos (Caligus sp) (Cuadro 1).

De los 150 especímenes muestreados, $92(61.3 \%)$ presentaron al menos un parásito, observándose mayor prevalencia en lisa $(86.7 \%)$, seguida de perico $(76.7 \%)$, mientras que se evidenció mayor carga parasitaria en lisa (424) y bonito (376) (Cuadro 2).

Se evidenció mayor frecuencia de las especies parasitarias Hepatoxylon trichiuri (56.7\%), Nybelinia sp (56.7\%) y Proleptus 
Cuadro 1. Prevalencia y lugar de ubicación de parásitos en 150 peces marítimos de interés comercial destinados al consumo humano, procedentes de dos terminales pesqueros en Lima Metropolitana (2014)

\begin{tabular}{|c|c|c|c|}
\hline Parásito & & $\begin{array}{c}\text { Prevalencia } \\
(\%)\end{array}$ & Lugar de ubicación \\
\hline Monogenea & Monocotile sp & 18.7 & Branquias \\
\hline \multicolumn{4}{|l|}{ Digenea } \\
\hline & Dinurus $s p$ & 2.7 & Intestinos \\
\hline Protozoo & Henneguya sp & 17.3 & Branquias, músculos \\
\hline \multirow[t]{4}{*}{ Cestodo } & Diphillobothrium pacificum & 4.7 & $\begin{array}{l}\text { Peritoneo visceral, } \\
\text { mú sculos }\end{array}$ \\
\hline & Hepatoxylon trichiuri & 61.3 & $\begin{array}{l}\text { Cavidad abdominal, } \\
\text { peritoneo visceral }\end{array}$ \\
\hline & Tentacularia coryphaenae & 22.7 & $\begin{array}{l}\text { Cavidad abdominal, } \\
\text { peritoneo visceral }\end{array}$ \\
\hline & Nybelinia sp & 14.0 & $\begin{array}{l}\text { Cavidad abdominal, } \\
\text { peritoneo visceral }\end{array}$ \\
\hline \multirow[t]{4}{*}{ Nematodo } & Anisakis simplex & 8.0 & $\begin{array}{l}\text { Intestinos, peritoneo } \\
\text { visceral }\end{array}$ \\
\hline & Anisakis physeteris & 2.7 & $\begin{array}{l}\text { Intestinos, peritoneo } \\
\text { visceral }\end{array}$ \\
\hline & Contracaecum sp & 2.7 & Intestinos \\
\hline & Proleptus sp & 28.7 & Intestinos \\
\hline \multicolumn{4}{|c|}{ Acantocéfalo } \\
\hline & Rhadinorhynchus sp & 13.3 & Intestinos \\
\hline Artrópodo & Caligus sp & 34.7 & Branquias \\
\hline
\end{tabular}

Cuadro 2. Prevalencia de parásitos y carga parasitaria en cinco especies de peces comerciales destinados al consumo humano procedentes de dos terminales pesqueros en Lima Metropolitana (2014) ( $\mathrm{n}=30$ por especie)

\begin{tabular}{lcc}
\hline Especie marina & $\begin{array}{c}\text { Prevalencia } \\
(\%)\end{array}$ & Carga parasitaria \\
\hline Bonito (Sarda chiliensis chiliensis) & 56.7 & 376 \\
Caballa (Scomber japonicus peruanus) & 23.3 & 64 \\
Jurel (Trachurus picturatus murphyi) & 63.3 & 140 \\
Lisa (Mugil cephalus) & 86.7 & 424 \\
Perico (Coryphaena hippurus) & 76.7 & 135 \\
\hline Total & 61.3 & - \\
\hline
\end{tabular}


Cuadro 3. Prevalencia (\%) de parásitos en cinco especies de peces comerciales destinados al consumo humano procedentes de dos terminales pesqueros en Lima Metropolitana ( $\mathrm{n}=30$ por especie)

\begin{tabular}{|c|c|c|c|c|c|}
\hline & Bonito & Caballa & Jurel & Lisa & Perico \\
\hline \multicolumn{6}{|l|}{ Monogenea } \\
\hline Monocotile sp & 20.0 & 20.0 & 26.7 & 13.3 & 13.3 \\
\hline \multicolumn{6}{|l|}{ Digenea } \\
\hline Dinurus sp & - & - & - & - & 13.3 \\
\hline \multicolumn{6}{|l|}{ Protozoo } \\
\hline Henneguya sp & 20.0 & 13.3 & 13.3 & 20.0 & 20.0 \\
\hline \multicolumn{6}{|l|}{ Cestodo } \\
\hline Diphyllobothrium pacificum & 20.0 & - & - & - & 3.3 \\
\hline Hepatoxylon trichiuri & 56.7 & 23.3 & 63.3 & 86.7 & 76.7 \\
\hline Tentacularia coryphaenae & 20.0 & - & 33.3 & 26.7 & 33.3 \\
\hline Nybelinia $\mathrm{sp}$ & 56.7 & - & 13.3 & - & - \\
\hline \multicolumn{6}{|l|}{ Nematodo } \\
\hline Anisakis simplex & 13.3 & 10.0 & 10.0 & - & 6.7 \\
\hline Anisakis physeteris & 3.3 & 3.3 & 3.3 & - & 3.3 \\
\hline Contracaecum sp & - & - & - & 13.3 & - \\
\hline Proleptus sp & 56.7 & - & - & 86.7 & - \\
\hline \multicolumn{6}{|l|}{ Acantocéfalo } \\
\hline Rhadinorhynchus sp & 23.3 & - & 43.3 & - & - \\
\hline \multicolumn{6}{|l|}{ Artrópodo } \\
\hline Caligus sp & 40.0 & 23.3 & 30.0 & 40.0 & 40.0 \\
\hline
\end{tabular}

sp (56.7\%) en bonito; Hepatoxylon trichiuri (23.3\%) y Caligus sp (23.3\%) en caballa; Hepatoxylon trichiuri $(63.3 \%) \quad \mathrm{y}$ Rhadinorhynchus sp (43.3\%) en jurel; Hepatoxylon trichiuri (86.7\%) y Caligus sp (40.0\%) en lisa; y Hepatoxylon trichiuri (76.7\%) y Caligus sp (40.0\%) en perico (Cuadro 3).

Dentro de las especies reconocidas como de importancia en salud pública, se observó la presencia de Anisakis sp (A. simplex y $A$. physeteris) en cuatro de las cinco espe- cies (lisa fue la excepción), mientras que $D$. pacificum fue solo observado en bonito $\mathrm{y}$ perico (Cuadro 3).

\section{Discusión}

La fauna parasitaria en las especies de peces marinos en el litoral peruano es variada y, conforme se producen cambios climatológicos, puede variar aún más, por lo que es importante determinar las especies parasitarias que la integran y su repercusión en la 


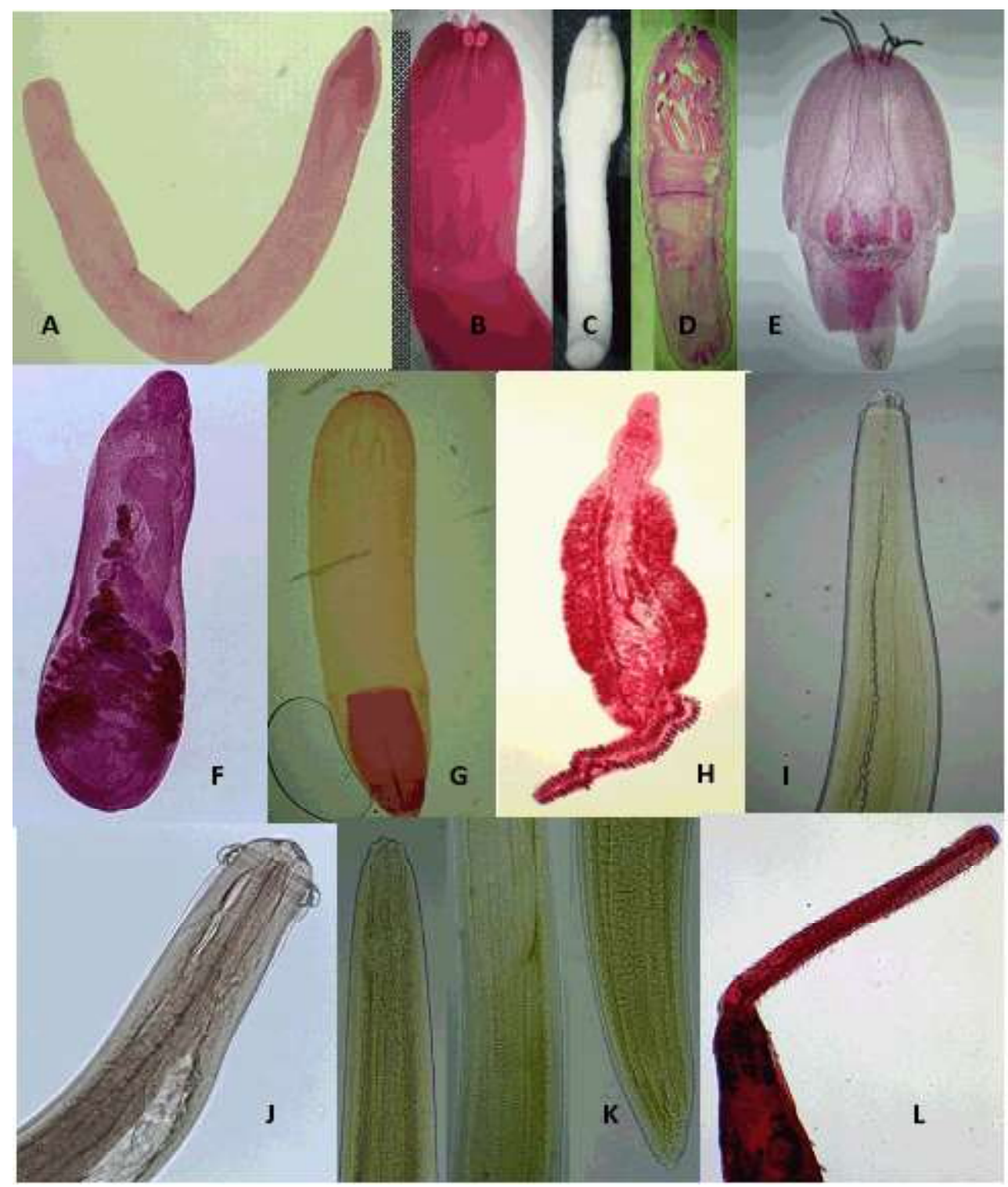

Figura 1. Parásitos hallados en peces marinos destinados al consumo humano en Lima Metropolitana. A: Plerocercoide de Diphillobothrium pacificum (10X); B, C, D: Hepatoxylon trichiuri (10X); E: Nybelinia sp (10X); F: Dinurus sp (10X); G: Tentacularia coryphaenae (10X); H: Microcotyle sp (10X); I: Contracaecum sp (10X); J: Proleptus sp (10X); K: Anisakis simplex (10X); L; Rhadinorhynchus sp (10X)

salud pública. Asimismo, si se considera el hábito alimenticio que tiene el poblador peruano de consumir productos pesqueros sin haber sido sometidos a un adecuado proceso de cocción (ejemplo, el cebiche) o sin haber tenido las condiciones higiénico-sanitarias adecuadas durante su preparación.
El presente estudio demostró la presencia de 13 especies de parásitos en una muestra de 150 especímenes que involucran a cinco especies de peces. Varios de estos parásitos han sido reportados en especies de peces marinos (Pérez et al., 1999; Llerena et al., 2001; Cabrera et al., 2002; Iannacone, 
2004; Aragort 2006; Iannacone y Alvariño, 2009; Vásquez-Ruiz y Jara-Campos, 2012; Chero et al., 2014).

Lisa (Mugil cephalus) presentó la mayor prevalencia y mayor carga parasitaria entre las cinco especies; sin embargo, aun cuando las especies de parásitos hallados, según la literatura científica, no representan un riesgo para la salud pública, se requiere mejorar la manipulación del producto pesquero durante su comercialización, ya que la presencia de parásitos en los peces acelera el proceso de descomposición del producto por las lesiones asociadas a la entrada de otros gérmenes como bacterias y hongos (Lamothe, 1994).

De las especies halladas de importancia en salud pública, $A$. simplex y $A$. physeteris no fueron encontrados en la lisa, especie marina en la cual se detectó Contracaecum sp, anisákido también reportado en esta especie en el país (Pérez et al., 1999; Iannacone y Alvariño, 2009; VásquezRuiz y Jara-Campos, 2012). D. pacificum fue detectado en bonito y perico, pero además se registra en jurel (Pérez et al., 1999) y caballa (Oliva et al., 2008). Es importante indicar que Iannacone y Alvariño (2009) mencionan la lisa como hospedador de Anisakis sp y que Diphyllobothrium sp tiene muy bajo potencial zoonótico en la costa central peruana.

La infección causada por parásitos de la Familia Anisakidae (Anisakis simplex, A. physeteris y Pseudoterranova decipiens) es denominada anisakiasis o anisakidosis. Los hospedadores definitivos son los mamíferos marinos (ballenas, delfines y lobos marinos) y los hospedadores intermediarios son los crustáceos, peces y calamares, donde se encuentra el estadio infectante del parásito - $\mathrm{L}_{3}$ (Alejo-Plata et al., 2003). La infección por el Diphyllobothrium sp se denomina difilobotriasis y es causada por las especies $D$. pacificum, D. latum, D. dendriticum, $D$. ursi, D. dalliae y D. klebanovskyii (Benenson, 1997). En el caso de $D$. pacificum los hospederos definitivos son los mamíferos marinos y los intermediarios son los crustáceos y peces, donde se encuentra el estadio infectante del parásito (Plerocercoide). En ambos casos, el hombre actúa como hospedador accidental cuando consume alimentos crudos o poco cocidos, como el «cebiche» o «tiradito» (Cabrera y Trillo-Altamirano, 2004). En Perú, se reportan casos de anisakidosis (Tantaleán y Huiza, 1993; Barriga et al., 1999; Cabrera et al., 2003) y de difilobotriasis en humanos (Medina et al., 2002).

\section{Conclusiones}

- Se reporta la presencia de 13 especies de parásitos en cinco especies de peces marinos de la costa central del Perú (Sarda chiliensis chiliensis [bonito], Scomber japonicus [caballa], Trachurus symmetricus murphyi [jurel], Mugil cephalus ([lisa] y Coryphaena hippurus [perico]), siendo un monogeneo, un digeneo, un protozoario, cuatro cestodos, cuatro nematodos, un acantocéfalo y un artópodo.

- $\quad$ El 61.3\% (92/150) de los peces presentaron al menos un parásito, observándose mayor prevalencia en lisa (86.67\%), seguida de perico $(76.67 \%)$. Asimismo, la mayor carga parasitaria se observó en lisa (424) y bonito (376).

- Los parásitos más frecuentes fueron Hepatoxylon trichiuri, Nybelinia sp y Proleptus sp.

- Dentro de los parásitos de importancia en salud pública se encontró Anisakis sp en bonito, caballa, jurel y perico, en tanto que Diphillobothrium pacificum en bonito y perico.

\section{Agradecimientos}

Los autores agradecen al Fondo Nacional de Desarrollo Científico, Tecnológico y de Innovación Tecnológica (FONDECYTCIENCIACTIVA) por el apoyo financiero 
brindado al Programa de Sanidad Acuícola (Contrato 230-2015 FONDECYT) de la $\mathrm{UPCH}$ «Proyecto: Identificación de agentes infecciosos en peces marinos y continentales del Perú del Concurso Programas de Maestría en Universidades Peruanas» que permitió los análisis y la capacitación técnica de nuestros investigadores para la ejecución del estudio.

\section{Literatura Citada}

1. Adis J, Arias J, Rueda G, Watthias K. 2006. Aquatic biodiversity in Latin America. Vol 1. $2^{\text {nd }}$ Ed. Sofia, Bulgaria: Pensoft. 509 p.

2. Alejo-Plata C, Cerdenares-Ladrón de Guevara G, Gonzales-Medina G. 2003. Larvas de Anisakis sp (Nematoda: Anisakidae), presentes en el pez dorado (Corhyphaena hippurus), en las costas de Oazaca, México. Viencia y Mar 7: 45-49.

3. Anderson R, Chabaud A, Willmott S. 2009. Keys to the nematode parasites of vertebrates. London, UK: CAB International. $480 \mathrm{p}$.

4. Aragort W. 2006. Parasitismo en peces de interés comercial y su repercusión en la salud pública. Ceniap Hoy N. ${ }^{\circ} 10$. [Internet]. Disponible en: http://www. ceniap.gov.ve/ceniaphoy/articulos/n10/ arti/aragort_w/arti/aragort_w.htm

5. Barriga J, Salazar F, Barriga E. 1999. Anisakiasis: presentación de un caso y revisión de la literatura. Rev Gastroenterol Peru 19: 317-323.

6. Benenson A. 1997. Manual para el control de enfermedades transmisibles. Publicaciones Científicas N. ${ }^{\circ}$ 564. Washington: OPS. $541 \mathrm{p}$.

7. Bush AO, Lafferty KD, Lotz JM, Shostak AW. 1997. Parasitology meets ecology on its own terms: Margolis et al. Revisited. J Parasitol 83: 575-583.

8. Cabrera R, Luna-Pineda M, SuárezOgnio L. 2003. Nuevo caso de infección humana por una larva de
Pseudoterranova decipiens (Nematoda, Anisakidae) en el Perú. Rev Gastroenterol Perú 23: 217-220.

9. Cabrera R, Suárez-Ognio L, Martínez $R$, Leiva R, Gambirazio C, Ruiz J. 2002. Larvas de Anisakis physeteris y otros helmintos en Coryphaena hippurus «perico» comercializados en el mercado pesquero de Ventanilla, Callao, Perú. Rev Peru Biol 9: 23-28. doi: 10.15381/rpb.v9i1.2517

10. Cabrera R, Trillo-Altamirano $M$. 2004. Anisakidosis: ¿Una zoonosis parasitaria marina desconocida o emergente en el Perú? Rev Gastroenterol Perú 24:335-342.

11. Chero J, Cruces C, Iannacone J, Saenz G, Alvariño L. 2014. Helmintos parásitos de Anisotremus scapularis (Tschudi, 1846) (Perciformes: Haemulidae) «chita» adquiridos en el terminal pesquero de Villa María del Triunfo, Lima, Perú. Neotrop Helminthol 8: 429438.

12. Eiras JC, Takemoto RM, Pavanelli $G C$. 2002. Métodos de estúdio y técnicas laboratoriales en parasitologia de peces. España: Ed Acribia. 142 p.

13. El-Deen N, Hady A, Shalaby S, Zaki M. 2012. Field Studies on Caligus disease among cultured Mugil cephalus in brackish water fish farms. Life Sci J 9(3): 733-737.

14. [FAO]. Origanización de las Nacionales Unidas para la Alimentación y la Agricultura. 2010. Perfíles de pesca y acuicultura por países. La República del Perú. [Internet]. Disponible en: http://www.fao.org/fishery/facp/PER/es

15. Iannacone J. 2004. Metazoos parásitos de la mojarrilla Stellifer minor (Tschudi) (Osteichthyes, Sciaenidae) capturados por pesquería artesanal en Chorrillos, Lima, Perú. Rev Brasileira Zool 21: 815-820. doi: 10.1590/S010181752004000400015

16. Iannacone J, Alvariño L. 2009. Metazoos parásitos de Mugil cephalus Linnaeus, 1758 (Mugilidae: Perciformes) procedentes del terminal pesquero de 
Chorrillos, Lima, Perú. Neotrop Helminthol 3: 15-27.

17. [INEI] Instituto Nacional de Estadística e Informática. 2014. Compendio estadístico Perú 2014. [Internet]. Disponible en: https://www.inei.gob.pe/media/ MenuRecursivo/publicaciones_digitales/ Est/Lib1173/compendio2014.html

18. Khalil L, Jones A, Bray RA. 1994. Keys to the cestode parasites of vertebrates. UK: CAB International. $768 \mathrm{p}$.

19. Lamothe R. 1994. Importancia de la helmintología en el desarrollo de la acuacultura. Anales Inst Biol Univ Nac Autón México Ser Zool 65: 195-200.

20. Llerena C, Chávez A, Casas E. 2001. Frecuencia de larvas diphyllobothriidae y larvas Anisakidae en peces marinos comerciales del Terminal Pesquero de Ventanilla-Callao. Rev Vet Inv Perú 12: 58-62. doi: 10.15381/rivep.v12i1.7425

21. Margolis L, Esch G, Holmes J, Kuris A, Schad G. 1982. The use of ecological terms in parasitology (report of an ad hoc committee of the American Society of Parasitologists). J Parasitol 68: 131-133.

22. Medina J, Tantaleán M, León M, Cano M. 2002. Diphyllobotrium pacificum en niños del Perú. Diagnostico 41: 161-164.
23. Oliva M, Valdivia I, Costa G, Freitas $N$, Pinheiro de Carvalho M, Sánchez L, Luque J. 2008. What can metazoan parasites reveal about the taxonomy of Scomber japonicus Houttuyn in the coast of South America and Madeira Islands? J Fish Biol 72: 545-554. doi: 10.1111/j.1095-8649.2007.01725.x

24. Perez I, Chávez A, Casas E. 1999. Presencia de formas parasitarias en peces comerciales del mar Peruano. Rev Vet Inv Peru 10: 34-38. doi: 10.15381/ rivep.v10i1.6613

25. Schmidt G. 1986. CRC handbook of tapeworm identification. Boca Raton, Florida: CRC Press. 678 p.

26. Tantaleán M, Huiza F. 1993. Nematode larvae with medical importance found in sea fish from the peruvian shore, with two records of human infections. Rev Peru Med Trop 7: 61-65.

27. Tantaleán M, Sánchez L, Gómez L, Huiza A. 2005. Acantocéfalos del Perú. Rev Peru Biol 12(1): 83-92.

28. Vásquez-Ruiz C, Jara-Campos C. 2012. Prevalencia e intensidad parasitaria en Coryphaena hippurus y Mugil cephalus (Teleostei) desembarcados en los puertos Salaverry y Paita (Perú). Sciéndo 15(1): 22-32. 